\title{
VLT-UVES abundance analysis of four giants in NGC $6553^{\star, \star \star}$
}

\author{
A. Alves-Brito ${ }^{1}$, B. Barbuy ${ }^{1}$, M. Zoccali ${ }^{2}$, D. Minniti ${ }^{2}$, S. Ortolani $^{3}$, V. Hill ${ }^{4}$, A. Renzini ${ }^{5}$, L. Pasquini ${ }^{6}$, E. Bica ${ }^{7}$, \\ R. M. Rich ${ }^{8}$, J. Meléndez ${ }^{9}$, and Y. Momany ${ }^{3}$ \\ 1 Universidade de São Paulo, IAG, Rua do Matão 1226, Cidade Universitária, São Paulo 05508-900, Brazil \\ e-mail: [abrito; barbuy] @astro.iag.usp.br \\ 2 Universidad Catolica de Chile, Department of Astronomy \& Astrophysics, Casilla 306, Santiago 22, Chile \\ e-mail: [dante;mzoccali]@astro.puc.cl \\ 3 Università di Padova, Dipartimento di Astronomia, Vicolo dell'Osservatorio 2, 35122 Padova, Italy \\ e-mail: sergio.ortolani@unipd.it \\ 4 Osservatorio Astronomico di Padova, Vicolo dell'Osservatorio 5, 35122 Padova, Italy \\ e-mail: alvio.renzini@oapd.inaf.it \\ 5 European Southern Observatory, Karl Schwarzschild Strasse 2, 85748 Garching bei München, Germany \\ e-mail: lpasquin@eso.org \\ ${ }^{6}$ European Southern Observatory, Karl Schwarzschild Strasse 2, 85748 Garching bei München, Germany \\ e-mail: [arenzini;lpasquin]@eso.org \\ 7 Universidade Federal do Rio Grande do Sul, Departamento de Astronomia, CP 15051, Porto Alegre 91501-970, Brazil \\ e-mail: bica@if.ufrgs.br \\ 8 UCLA, Department of Physics \& Astronomy, 8979 Math-Sciences Building, Los Angeles, CA 90095-1562, USA \\ e-mail: rmr@astro.ucla.edu \\ 9 Australian National University, Australia \\ e-mail: jorge@mso.anu.edu.au
}

Received 24 April 2006 / Accepted 10 August 2006

\section{ABSTRACT}

Context. Metal-rich globular clusters trace the formation of bulges. Abundance ratios in the metal-rich globular clusters such as NGC 6553 can constrain the formation timescale of the Galactic bulge.

Aims. The purpose of this study is to determine the metallicity and elemental ratios in individual stars of the metal-rich bulge globular cluster NGC 6553.

Methods. A detailed abundance analysis of four giants in NGC 6553 is carried out, based on optical high-resolution échelle spectra obtained with UVES at the ESO VLT-UT2 Kueyen telescope.

Results. A metallicity $[\mathrm{Fe} / \mathrm{H}]=-0.20$ dex is derived, together with $\alpha$-element enhancement of $\mathrm{Mg}$ and $\mathrm{Si}([\mathrm{Mg} / \mathrm{Fe}]=+0.28,[\mathrm{Si} / \mathrm{Fe}]=$ $+0.21)$, solar $\mathrm{Ca}$ and $\mathrm{Ti}([\mathrm{Ca} / \mathrm{Fe}]=+0.05,[\mathrm{Ti} / \mathrm{Fe}]=-0.01)$, and a mild enhancement of the r-process element $\mathrm{Eu} \mathrm{with}[\mathrm{Eu} / \mathrm{Fe}]=+0.10$. A mean heliocentric radial velocity of $-1.86 \mathrm{~km} \mathrm{~s}^{-1}$ is measured. We compare our results with previous investigations of the cluster.

Key words. stars: abundances - globular clusters: individual: NGC 6553 - Hertzsprung-Russell (HR) and C-M diagrams Galaxy: bulge

\section{Introduction}

Metal-rich red globular clusters trace the build up of bulges and possibly of disks (Brodie \& Strader 2006). They may be formed during an early collapse (Forbes et al. 1997; Côté et al. 1998); otherwise, in hierarchical scenarios the metal-rich globular clusters would be direct probes of the merging events and assembly of the host galaxy (Ashman \& Zepf 1992; Beasley et al. 2002; Bekki 2005). Another possibility is bulge formation via the secular evolution of a bar (Kormendy \& Kennicutt 2004). Their detailed study should help constrain models of globular cluster formation in the early Galaxy.

Several studies have tried to infer some of the main properties of NGC 6553. Minniti (1995) argues that the metal-rich

\footnotetext{
* Observations collected both at the European Southern Observatory, Paranal, and La Silla, Chile (ESO programmes 65.L-0340, 65.L-0371, 67.D-0489, and 69.D-0582).

$\star \star$ Appendices are only available in electronic form at http://www. aanda.org
}

globular clusters in the inner spheroid are associated with the galactic bulge. Zoccali et al. $(2001,2003)$ determined the proper motion of NGC 6553 and conclude that this cluster follows the mean rotation of both disk and bulge stars, whereas Dinescu et al. (2003) conclude that this cluster belongs to a rotationally supported disk system. Ortolani et al. (1995) show that NGC 6528 and NGC 6553 are old. Another tool for better understanding the origin of these metal-rich clusters is the chemical composition of individual member stars.

The globular clusters in the bulge NGC 6553, NGC 6528, NGC 6440, and Liller 1 are among the most metal-rich clusters in the Galaxy (Barbuy et al. 1998). Ortolani et al. (1990) point out the turnover and fainter red giant branch (RGB) of NGC 6553, relative to less metal-rich clusters, indicating that TiO blanketing should contribute to the RGB behaviour, being in itself an indicator of high metallicity. These metal-rich clusters constitute the only available calibrators for metallicity and abundance ratio measurement methods based on low-resolution 
spectra and/or population synthesis of composite spectra of star clusters and galaxies.

NGC 6553 is the most studied bulge globular cluster, and yet only few stars were analysed at high resolution. The CCD analyses were carried out for one giant by Barbuy et al. (1992) and two giants by Barbuy et al. (1999) at moderate resolution $(R \sim$ 20000 ), five red horizontal branch stars by Cohen et al. (1999) at high resolution $(R \sim 34000)$, whereas in the $H$ band two giants were analysed by Origlia et al. (2002) at moderate resolution ( $R \sim 25000)$ and five giants by Meléndez et al. (2003) at high resolution $(R \sim 50000)$. Carretta et al. (2001) revised the Cohen et al. (1999) analysis. These previous results gave metallicities in the range $-0.55<[\mathrm{Fe} / \mathrm{H}]<-0.06$.

We present the analysis of four giants based on highresolution $(R=55000)$ spectra obtained at the VLT-UT2 Kueyen telescope equipped with the UVES spectrograph. These are the highest resolution observations available for stars in this cluster. Since we are dealing with cool metal-rich giants, high-resolution spectroscopy is mandatory.

The observations and radial velocity derivation are described in Sect. 2. In Sect. 3 the stellar parameters are presented, the abundances are derived in Sect. 4, the results are discussed in Sect. 5, and a summary is given in Sect. 6.

\section{Observations}

\subsection{Imaging}

NGC $6553\left(\alpha_{\mathrm{J} 2000}=18^{\mathrm{h}} 09^{\mathrm{m}} 17.6^{\mathrm{s}}, \delta_{\mathrm{J} 2000}=-25^{\mathrm{o}} 54^{\prime} 31^{\prime \prime}, l=\right.$ $\left.5.25^{\circ}, b=-3.03^{\circ}\right)$ is located at low galactic latitude and is projected in the direction of the Galactic centre with $X=+5.9 \mathrm{kpc}$, $Y=+0.5 \mathrm{kpc}$, and $Z=-0.3 \mathrm{kpc}$. It has a relatively low extinction of $E_{B-V}=0.70$ mag (Guarnieri et al. 1998) and a high luminosity of $M_{V}=-7.77 \mathrm{mag}$ (Harris 1996) ${ }^{1}$.

The $B V I$ observations of NGC 6553 were obtained in June 2002 with the wide-field imager (WFI) at the $2.2 \mathrm{~m}$ ESO-MPI telescope (La Silla, Chile). The data were obtained within our program dedicated to surveying the Galactic globular clusters with the WFI (Zoccali et al. 2001). The $J H K_{\mathrm{S}}$ colours are from the 2MASS Atlas (Cutri et al. 2003) ${ }^{2}$. No $J, H, K$ photometry for the sample stars II-64 and 267092 are currently available.

In Fig. 1 we show the location of target sample stars on the colour-diagram magnitude (CMD) of NGC 6553 using Hubble Space Telescope (HST) data (Ortolani et al. 1995), with three stars located at the horizontal branch (HB) level and one of them at the tip of the RGB. The identifications follow the notation given in Hartwick (1975) and in the WFI data obtained with the ESO-MPI telescope.

\subsection{High-resolution spectra}

High-resolution spectra of four giants in NGC 6553, in the wavelength range $\lambda \lambda$ 4800-6800 $\AA$, were obtained with the UVES spectrograph (Dekker et al. 2000) at the ESO VLT-UT2 Kueyen telescope. The red portion of the spectrum (5800-6800 ̊) was obtained with the MIT backsideilluminated ESO CCD \# 20, of $4096 \times 2048$ pixels, and pixel size $15 \times 15 \mu \mathrm{m}$. The blue portion of the spectrum (4800-5800 A) used ESO Marlene EEV CCD-44, backside illuminated, $4102 \times 2048$ pixels, and pixel size $15 \times 15 \mu \mathrm{m}$. With the UVES standard setup 580, the resolving power $(R=\lambda / \Delta \lambda)$

\footnotetext{
${ }^{1}$ http://www.physics.mcmaster.ca/Globular.html

${ }^{2}$ http://ipac.caltech.edu./2mass/releases/allsky/
}

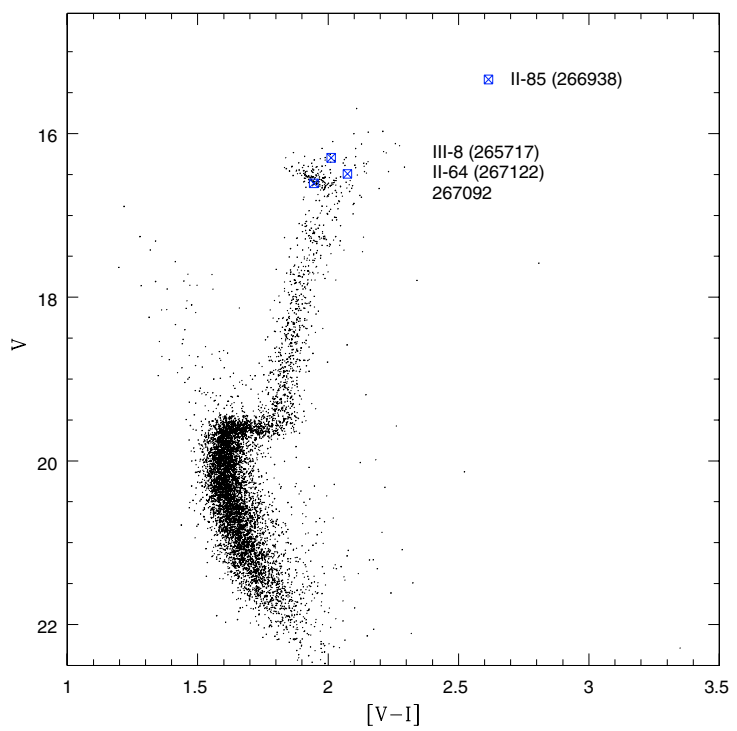

Fig. 1. $V$ vs. $V-I$ CMD of NGC 6553 with the target stars indicated.

is $R \simeq 55000$ for a slit of $0.8^{\prime \prime}$. Typical signal-to-noise $(S / N)$ ratios for the spectra were obtained considering average values at different wavelengths. The pixel scale is $0.0147 \AA /$ pix. Spectra of rapidly rotating hot B stars at similar airmasses as the target were also observed, in order to correct for telluric lines. Table 1 shows the log of observations.

The spectra were reduced using the UVES context of the MIDAS reduction package, including bias and inter-order background subtraction, flatfield correction, extraction and wavelength calibration (Ballester et al. 2000). The stars were observed in pairs, II-64 together with II-85 and III-8 with 267092 , and reduced taking this into account. In Fig. 2 typical sample spectra are shown.

\subsection{Radial velocities}

The radial velocities $v_{\mathrm{r}}$ were measured with the automatic code DAOSPEC (Stetson \& Pancino 2006, in preparation) based on line shifts of a given list of wavelengths in the range $5800<$ $\lambda<6800 \AA$. The heliocentric radial velocities $v_{\mathrm{r}}^{\mathrm{h}}$ were determined using the IRAF task rvcorrect. With these methods, the standard errors of the average velocities are $\sim 0.5 \mathrm{~km} \mathrm{~s}^{-1}$. A mean $v_{\mathrm{r}}=-0.27 \pm 1.99(\sigma=3.98,4$ stars $) \mathrm{km} \mathrm{s}^{-1}$ or heliocentric $v_{\mathrm{r}}^{\text {hel }}=-1.86 \pm 2.01(\sigma=4.02,4$ stars $) \mathrm{km} \mathrm{s}^{-1}$ was found for NGC 6553. Combining high-resolution radial velocities of stars from the present work and from Origlia et al. (2002), Barbuy et al. (1999), Cohen et al. (1999), and Meléndez et al. (2003), a mean value of $v_{\mathrm{r}}^{\mathrm{h}}=+1.30 \pm 1.41\left(\sigma=6.50 \mathrm{~km} \mathrm{~s}^{-1}\right)$ was found. This result is in good agreement with the value of $-1 \mathrm{~km} \mathrm{~s}^{-1}$, based on different methods and from low-resolution spectra of Coelho et al. (2001), and the value $8.4(\sigma=+8.4$, 21 stars) $\mathrm{km} \mathrm{s}^{-1}$ derived by Rutledge et al. (1997).

\section{Stellar parameters}

\subsection{Photometric parameters}

Adopting a reddening $E_{B-V}=0.70$ for NGC 6553 (Guarnieri et al. 1998; Sagar et al. 1999; Meléndez et al. 2003) and reddening ratios $E_{V-I} / E_{B-V}=1.33$ (Dean et al. 1978), $E_{V-K} / E_{B-V}=2.744$, and $E_{J-K} / E_{B-V}=0.52$ (Rieke \& Lebofsky 1985), we obtained dereddened colours to derive photometric 
Table 1. Log of spectroscopic observations. The $S / N$ ratio is given per resolution element (7 pixels); the value per pixel is the value per resolution element divided by $\sim 2.8$.

\begin{tabular}{ccccccccc}
\hline \hline Star & $\begin{array}{c}\alpha_{\text {J2000 }} \\
{[\mathrm{h} \mathrm{m} \mathrm{s}]}\end{array}$ & $\begin{array}{c}\delta_{\text {J2000 }} \\
{[\mathrm{d} \mathrm{m} \mathrm{s}]}\end{array}$ & Date & UT & Exp & Seeing & Airmass & $(S / N) /$ res. el. \\
& $18: 09: 18.31$ & $-25: 55: 01.15$ & 2000 June 26 & $14: 21: 25.00$ & $2 \times 3600$ & 0.8 & 1.4 & 110 \\
\hline II-64 267122 & $18: 4$ & “ & \\
II-85 266938 & $18: 09: 17.96$ & $-25: 55: 04.89$ & 2000 June 26 & $14: 21: 25.00$ & $2 \times 3600$ & 0.8 & 1.02 & 170 \\
III-8 265717 & $18: 09: 13.05$ & $-25: 55: 30.00$ & 2000 June 26 & $17: 37: 49.00$ & $2 \times 5400$ & 0.8 & 1.02 & 110 \\
\hline
\end{tabular}



Fig. 2. Typical raw spectra of NGC 6553 with some atomic lines identified. In each panel the heliocentric radial velocity is given.

temperatures. The 2MASS $K_{\mathrm{S}}$ magnitudes were transformed to the TCS (Telescopio Carlos Sánchez) system using relations by Carpenter (2001) and Alonso et al. (1998). Effective photometric temperatures were then determined based on the infrared flux method (IRFM) using the empirical transformations of Alonso et al. (1999, 2001, hereafter AAM99), where metallicity values provided by Meléndez et al. (2003) were initially adopted. If we take into account the $1 \sigma$ uncertainties provided by AAM99 on $V-I\left(\sigma_{V-I}=125 \mathrm{~K}\right), V-K\left(\sigma_{V-K}=40 \mathrm{~K}\right)$, and $J-K$ $\left(\sigma_{J-K}=125 \mathrm{~K}\right)$, the uncertainty in the photometric temperatures is about $130 \mathrm{~K}$. Table 2 lists the photometric data for the programme stars, together with the temperature estimated from each colour. The final photometric temperature for each star was averaged from these individual values.

The surface gravities $\log g$ were derived with the classical relation

$\log \left(\frac{g_{*}}{g_{\odot}}\right)=+4 \log \left(\frac{T_{*}}{T_{\odot}}\right)+0.4\left(M_{\mathrm{bol}}^{*}-M_{\mathrm{bol}}^{\odot}\right)+\log \left(\frac{M_{*}}{M_{\odot}}\right)$

by adopting $T_{\odot}=5780 \mathrm{~K}, M_{\mathrm{bol}^{\odot}}=4.75, \log g_{\odot}=4.44 \mathrm{dex}$, and $M_{*}=0.80 M_{\odot}$. To determine $M_{\text {bol }}^{*}$ we assume a distance modulus of $(m-M)_{0}=13.60$, visual selective-to-total extinction $A_{\mathrm{V}}=$ 2.17, and bolometric correction $B C_{V}$ values from AAM99.

We adopt errors due to uncertainties in the photometric effective temperature of $T_{\text {eff }}\left(\sigma_{T_{\text {eff }}}=130 \mathrm{~K}\right.$, according to AAM99) and in the stellar mass of $M_{*}\left(\sigma_{M_{*}}=0.2 M_{\odot}\right)$. The error in $M_{\mathrm{bol}}=$ $\left(M_{\mathrm{V}}-A_{\mathrm{V}}\right)+\mathrm{BC}(\mathrm{V})$ is mostly due to the $M_{\mathrm{V}}$ value and total extinction $A_{\mathrm{V}}$, the latter with an uncertainty around \pm 0.05 mag. For $M_{\mathrm{V}}$, adopting an error in distance as large as $20 \%$, we get
Table 2. Photometric effective temperatures $T_{\text {eff }}$ derived using $V-I$, $V-K$, and $J-K$ based on relations from AAM99. $(V-I)_{0}^{\mathrm{C}}$ and $(V-I)_{0}^{\mathrm{J}}$ refer to reddening-corrected Johnson-Cousins and Johnson photometric systems.

\begin{tabular}{lcccc}
\hline \hline Parameter & II-64 & II-85 & III-8 & 267092 \\
\hline \multicolumn{5}{c}{ Magnitudes and Colours } \\
$V[\mathrm{mag}]$ & 16.492 & 15.339 & 16.297 & 16.608 \\
$I[\mathrm{mag}]$ & 14.418 & 12.724 & 14.285 & 14.663 \\
$J[\mathrm{mag}]$ & - & 10.979 & 12.798 & - \\
$H[\mathrm{mag}]$ & - & 9.975 & 11.983 & - \\
$K_{\mathrm{S}}[\mathrm{mag}]$ & - & 9.647 & 11.749 & - \\
$(V-I)_{0}^{\mathrm{C}}$ & 1.143 & 1.684 & 1.081 & 1.014 \\
$(V-I)_{0}^{9}$ & 1.469 & 2.172 & 1.389 & 1.303 \\
\hline \multicolumn{5}{c}{ Effective Temperatures } \\
$T_{V-I}[\mathrm{~K}]$ & 4448 & 3773 & 4564 & 4700 \\
$T_{V-K}[\mathrm{~K}]$ & - & 3853 & 4500 & - \\
$T_{J-K}[\mathrm{~K}]$ & - & 3901 & 4564 & - \\
$T_{\text {mean }}[\mathrm{K}]$ & 4448 & 3842 & 4543 & 4700 \\
\hline
\end{tabular}

$\sigma_{M_{\text {bol }}} \sim 0.2 \mathrm{mag}$, and errors on the adopted photometric gravities of \pm 0.2 dex.

\subsection{Spectroscopic parameters}

\subsubsection{Equivalent widths, oscillator strengths, and damping constants}

Equivalent widths $W_{\lambda}$ of Fe I and Fe II lines were measured using the automatic code DAOSPEC developed by Stetson \& Pancino (2006, in preparation). Given a reference line list and a first estimate of FWHM by an iterative process, DAOSPEC fits a continuum and measures $W_{\lambda}$ by fitting a Gaussian profile of fixed width. The overall uncertainty is $<10 \%$. A comparison of $W_{\lambda} \mathrm{s}$ measured with both IRAF and DAOSPEC was presented in Fig. 3 of Alves-Brito et al. (2005). Lines with $10<W_{\lambda}<150 \mathrm{~m} \AA$ were selected.

The oscillator strengths $\log g f$ of Fe I lines were taken from the National Institute of Standards \& Technology (NIST) atomic database (Martin et al. 2002), while those of the Fe II lines were adopted according to the renormalized values from Meléndez \& Barbuy (2002, 2006, in preparation) and Meléndez et al. (2006). The Ba II 6141, 6496, La II 6390, and Eu II $6645 \AA$ lines show a hyperfine splitting structure, and those were taken into account using data from Biehl (1976) and Lawler (2001), together with solar isotopic ratios. Damping constants and oscillator strengths for the elements other than Fe were adopted from Barbuy et al. (2006). Solar abundances were adopted from Grevesse \& Sauval (1998).

\subsubsection{Derivation of spectroscopic parameters}

The effective temperatures were then checked by imposing an excitation equilibrium on Fe I and Fe II lines of different 
Table 3. Photometric and adopted spectroscopic stellar parameters.

\begin{tabular}{|c|c|c|c|c|c|c|}
\hline Star & $\begin{array}{l}M_{\text {bol }} \\
\text { mag }\end{array}$ & $\begin{array}{c}T_{\text {eff }} \\
\mathrm{K}\end{array}$ & $\begin{array}{c}v_{t} \\
\mathrm{~km} \mathrm{~s}^{-1}\end{array}$ & $\begin{array}{c}\log g^{a} \\
\operatorname{dex}\end{array}$ & $\begin{array}{c}\mathrm{Fe} \mathrm{I} / \mathrm{H}] \\
\operatorname{dex}\end{array}$ & $\begin{array}{c}\mathrm{Fe} \mathrm{II} / \mathrm{H}] \\
\operatorname{dex}\end{array}$ \\
\hline \multicolumn{7}{|c|}{ Photometric } \\
\hline II-64 267122 & +0.1987 & 4448 & 1.45 & 2.07 & $-0.23 \pm 0.15$ & $-0.10 \pm 0.03$ \\
\hline II-85 266938 & -1.6190 & 3842 & 1.38 & 1.08 & $-0.23 \pm$ & $-0.29 \pm 0.09$ \\
\hline III-8 265717 & +0.0653 & 4543 & 1.32 & 2.05 & $-0.21 \pm 0.16$ & $-0.23 \pm 0.09$ \\
\hline 267092 & +0.4672 & 4700 & 1.50 & 2.27 & $-0.19 \pm 0.11$ & $-0.46 \pm 0.06$ \\
\hline \multicolumn{7}{|c|}{ Spectroscopic } \\
\hline II-64 267122 & - & 4500 & 1.45 & 2.20 & $-0.20 \pm 0.15$ & $-0.20 \pm 0.01$ \\
\hline II-85 266938 & - & 3800 & 1.38 & 1.10 & $-0.23 \pm 0.15$ & $-0.29 \pm 0.09$ \\
\hline III-8 265717 & - & 4600 & 1.40 & 2.40 & $-0.17 \pm 0.15$ & $-0.17 \pm 0.04$ \\
\hline 267092 & - & 4600 & 1.50 & 2.50 & $-0.21 \pm 0.12$ & $-0.22 \pm 0.06$ \\
\hline
\end{tabular}
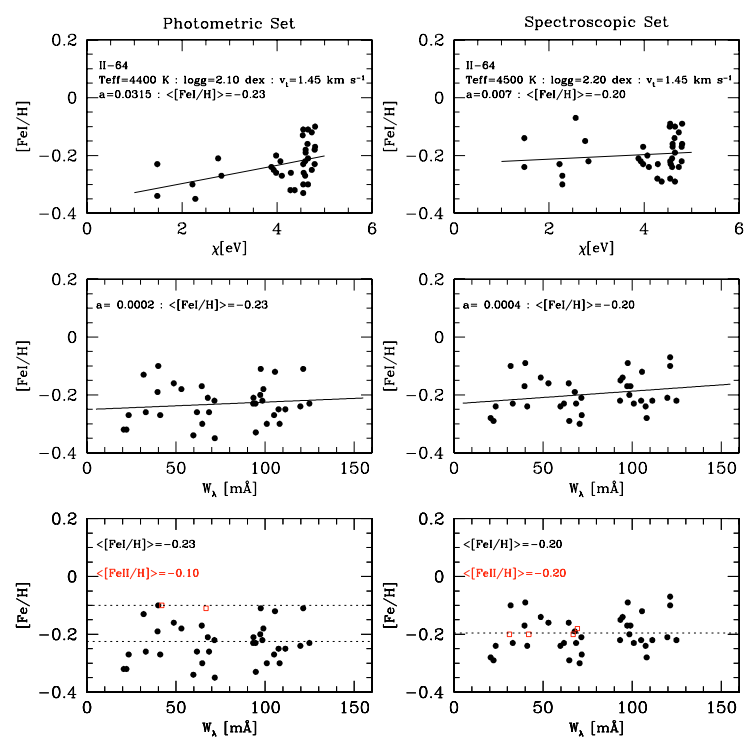

Fig. 3. Demonstration of the offset in temperature, surface gravity, and metallicity found for the star II-64 based on photometric and spectroscopic methods. The constant a is the slope of the least-squares fit to the data. Top: Fe I vs. $\chi$. Middle: Fe I vs. $W_{\lambda}$. Bottom: Fe I (circles) and Fe II (squares) vs. $W_{\lambda}$. The dotted line corresponds to the mean values found using only those measured within $1 \sigma$, as employed by the $\sigma$ clipping method.

excitation potentials. The Fe I and Fe II lines employed in this analysis are listed in Table A.2, together with their equivalent widths for the sample stars. Hereafter we refer to the atmospheric parameters $\left[T_{\text {eff }}, v_{t}, \log g\right]$ obtained by imposing constraints on Fe I and/or Fe II abundances as spectroscopic parameters. A change of $100 \mathrm{~K}$ on $T_{\text {eff }}$ causes a recognisable trend in the plane Fe I abundance versus excitation potential, such that this can be considered as a reasonable uncertainty value. The microturbulent velocities $v_{t}$ were determined using Fe I lines. The uncertainty derived from the Fe I abundance versus $W_{\lambda}$ is $0.2 \mathrm{~km} \mathrm{~s}^{-1}$.

The spectroscopic gravity $\log g$ was derived from ionization equilibrium of Fe I and Fe II lines. We found a mean offset between the spectroscopic and photometric (Sect. 3.2.1) values of $\Delta \log g$ (spectro - photo $)=+0.17 \pm 0.08(\sigma=+0.17$, 4 stars). However, this procedure is affected by several uncertainties, amounting to an upper limit of $\pm 0.30 \mathrm{dex}$. This value corresponds to a difference higher than $1 \sigma$ in the value of $[\mathrm{Fe} \mathrm{II/H].} \mathrm{In}$ Fig. 3 an example of determination of the atmospheric parameters for the star II-64 is shown.

\subsection{Final parameters and metallicity}

To derive metallicities, Fe I and Fe II lines with $15<W_{\lambda}<$ $150 \mathrm{~m} \AA$ and $\lambda>5800 \AA$ were selected. Photospheric 1D models for the sample giants were extracted from the NMARCS grid (Plez et al. 1992).

The LTE abundance analysis and the spectrum synthesis calculations were performed using the codes by Spite (1967) and subsequent improvements in the last thirty years, described in Cayrel et al. (1991) and Barbuy et al. (2003).

Stellar parameters were derived by initially adopting the photometric effective temperature and gravity and then further constraining the temperature by imposing excitation equilibrium for Fe I lines and the gravity by imposing ionization equilibrium for Fe I and Fe II, as described in previous sections. The entire process of defining the atmospheric parameters is iterated until a consistent set of model atmosphere parameters is finally obtained. Table 3 summarises the derived atmospheric parameters. We adopt the spectroscopic parameters for the subsequent analysis, having used the photometric parameters only as initial values; therefore, the uncertainties on the reddening described in Sect. 3.1 do not affect the final results. We point out otherwise that the good agreement between the spectroscopic and photometric parameters indicates that the reddening value adopted is satisfactory.

The mean offset in the $T_{\text {eff }}$ values is $25 \pm 48 \mathrm{~K}(\sigma=96$, 4 stars) in the sense of spectroscopic minus photometric temperature. This means that the mean $T_{\text {eff }}$ based on photometry is $\sim 25 \mathrm{~K}$ cooler than the spectroscopic ones. Along the iterative process of $[\mathrm{Fe} / \mathrm{H}]$ determination, discrepant lines were clipped out within $1 \sigma$ of the mean. Tests on the sample stars showed that a $2 \sigma$ clipping gives differences of $[\mathrm{Fe} / \mathrm{H}]< \pm 0.01$. A mean metallicity of $[\mathrm{Fe} / \mathrm{H}]=-0.20 \pm 0.01(\sigma=0.02,4$ stars $)$ is found for the sample stars. The spectroscopic parameters were adopted for the abundance analysis.

\section{Spectrum synthesis}

Abundance ratios were derived for the following eleven elements $\mathrm{Na}, \mathrm{Mg}, \mathrm{Al}, \mathrm{Si}, \mathrm{Ca}, \mathrm{Ti}, \mathrm{Fe}, \mathrm{Zr}, \mathrm{Ba}, \mathrm{La}$, and Eu. Most lines used are the same reported in Barbuy et al. (2006). Table A.1 gives the relevant parameters used in the spectrum synthesis of each line and resulting abundances.

Elemental abundances were obtained through line-by-line spectrum synthesis calculations with atomic lines as described in Sect. 3 and molecular lines of $\mathrm{CN} \mathrm{A}^{2} \Pi-\mathrm{X}^{2} \Sigma, \mathrm{C}_{2}$ Swan $\mathrm{A}^{3} \Pi-\mathrm{X}^{3} \Pi$ and TiO $\mathrm{A}^{3} \Phi-\mathrm{X}^{3} \Delta \gamma$ and $\mathrm{B}^{3} \Pi-\mathrm{X}^{3} \Delta \gamma^{\prime}$ systems taken into account. Table 4 gives the final mean results for each star, where 
Table 4. Mean abundance ratios of the programme stars and final mean cluster abundances derived.

\begin{tabular}{|c|c|c|c|c|c|c|c|c|c|c|}
\hline \multirow[b]{2}{*}{ Species } & \multirow[b]{2}{*}{$\epsilon_{\odot}(\mathrm{X})^{a}$} & \multicolumn{2}{|r|}{ II-64 } & \multicolumn{2}{|r|}{ II-85 } & \multicolumn{2}{|r|}{ III-8 } & \multicolumn{2}{|r|}{267092} & \multirow{2}{*}{$\begin{array}{c}\text { mean } \\
{[\mathrm{X} / \mathrm{Fe}]}\end{array}$} \\
\hline & & $N$ & {$[\mathrm{X} / \mathrm{Fe}]$} & $N$ & {$[\mathrm{X} / \mathrm{Fe}]$} & $N$ & {$[\mathrm{X} / \mathrm{Fe}]$} & $N$ & {$[\mathrm{X} / \mathrm{Fe}]$} & \\
\hline \multicolumn{11}{|c|}{$\alpha$-elements } \\
\hline Mg I & 7.58 & 3 & $+0.32 \pm 0.09$ & 3 & +0.27 & 3 & $+0.29 \pm 0.03$ & 3 & $+0.22 \pm 0.09$ & $+0.28 \pm 0.04$ \\
\hline $\mathrm{Si}$ I & 7.55 & 6 & $+0.27 \pm 0.09$ & 4 & $+0.22 \pm 0.08$ & 6 & $+0.17 \pm 0.07$ & 6 & $+0.17 \pm 0.03$ & $+0.21 \pm 0.05$ \\
\hline $\mathrm{CaI}$ & 6.36 & 15 & $+0.11 \pm 0.12$ & 14 & $+0.12 \pm 0.09$ & 15 & $+0.05 \pm 0.14$ & 15 & $-0.07 \pm 0.13$ & $+0.05 \pm 0.09$ \\
\hline Ti I & 5.02 & 15 & $+0.04 \pm 0.08$ & 15 & $+0.15 \pm 0.11$ & 15 & $-0.05 \pm 0.10$ & 15 & $-0.17 \pm 0.15$ & $-0.01 \pm 0.14$ \\
\hline Ti II & 5.02 & 2 & $+0.06 \pm 0.04$ & 2 & $+0.01 \pm 0.11$ & 2 & $-0.04 \pm 0.04$ & 2 & -0.07 & $-0.02 \pm 0.06$ \\
\hline \multicolumn{11}{|c|}{ Odd-Z elements } \\
\hline $\mathrm{Na} \mathrm{I}$ & 6.33 & 2 & +0.12 & 3 & +0.34 & 2 & -0.08 & 2 & +0.12 & $+0.16 \pm 0.23$ \\
\hline $\mathrm{Al} \mathrm{I}$ & 6.47 & 2 & $+0.25 \pm 0.03$ & - & - & 2 & $+0.15 \pm 0.17$ & 2 & +0.13 & $+0.18 \pm 0.06$ \\
\hline \multicolumn{11}{|c|}{ Heavy elements } \\
\hline $\mathrm{ZrI}$ & 2.60 & 1 & -0.74 & 1 & -0.64 & 1 & -0.64 & 1 & -0.64 & $-0.67 \pm 0.05$ \\
\hline Ba II & 2.13 & 2 & -0.08 & 2 & $-0.18 \pm 0.14$ & 2 & $-0.48 \pm 0.14$ & 2 & -0.38 & $-0.28 \pm 0.18$ \\
\hline La II & 1.17 & 1 & +0.01 & 1 & -0.22 & - & - & - & 一 & $-0.11 \pm 0.16$ \\
\hline Eu II & 0.51 & 1 & +0.09 & 1 & +0.09 & 1 & +0.10 & 1 & +0.14 & $+0.10 \pm 0.02$ \\
\hline \multicolumn{11}{|c|}{$[\mathrm{Fe} / \mathrm{H}]$} \\
\hline $\mathrm{Fe} I$ & 7.50 & 56 & $-0.20 \pm 0.15$ & 52 & $-0.23 \pm 0.15$ & 59 & $-0.17 \pm 0.15$ & 57 & $-0.21 \pm 0.12$ & $-0.20 \pm 0.02$ \\
\hline Fe II & 7.50 & 4 & $-0.20 \pm 0.01$ & 2 & $-0.29 \pm 0.09$ & 3 & $-0.17 \pm 0.04$ & 4 & $-0.22 \pm 0.06$ & $-0.22 \pm 0.05$ \\
\hline
\end{tabular}

${ }^{a}$ Solar abundances are from Grevesse \& Sauval (1998).

the abundances were normalized to Fe I for neutral elements and Fe II for ionized ones.

Abundances of the $\alpha$-elements $\mathrm{Mg}, \mathrm{Ca}, \mathrm{Si}$, and $\mathrm{Ti}$ were derived. The oxygen forbidden line at $6300 \AA$ blended with the strong sky emission line, given the low radial velocity of the cluster (Sect. 2.3), cannot be studied. In a previous work, Meléndez et al. (2003) derived the oxygen abundance of 5 giants in NGC 6553, based on $\mathrm{OH}$ infrared lines. We adopted the carbon, nitrogen, and oxygen abundances from that work in the present calculations, namely $[\mathrm{C} / \mathrm{Fe}]=-0.7,[\mathrm{~N} / \mathrm{Fe}]=+1.30$, $[\mathrm{O} / \mathrm{Fe}]=+0.25$. Figures 4 and 5 show the magnesium triplet at $6318.7,6319.2,6319.4 \AA$, and SiI 5948.548 $\AA$ in the giant II-85. Figure 6 shows the Europium line at $6645.127 \AA$ in the same star.

\subsection{Errors in abundance ratios}

Abundances for each species given in Table 4 were obtained by averaging the abundances resulting from all measured lines. Consequently, we give the $1 \sigma$ scatter (standard deviation) of each measurement, such that the standard error can be obtained easily.

The uncertainties on atmospheric parameters were discussed in Sect. 3. To estimate the uncertainties on the derivation of abundances due to the choice of stellar parameters, we show the sensitivity of the abundances in Table 5 for the star II- 64 by varying the temperature by $100 \mathrm{~K}$, surface gravity by $+0.30 \mathrm{dex}$, and microturbulent velocity by $+0.20 \mathrm{~km} \mathrm{~s}^{-1}$, which are typical errors in our analysis. The total error is given in the last column as the quadratic sum of all uncertainties. We can see that the total uncertainties are lower than $0.21 \mathrm{dex}$. In the next sections these uncertainties correspond to the $1 \sigma$ scatter for each atomic species.

\subsection{Comparison with previous work}

In the present work a metallicity of $[\mathrm{Fe} / \mathrm{H}]=-0.20$ is found for NGC 6553. A derivation based on photometric parameters

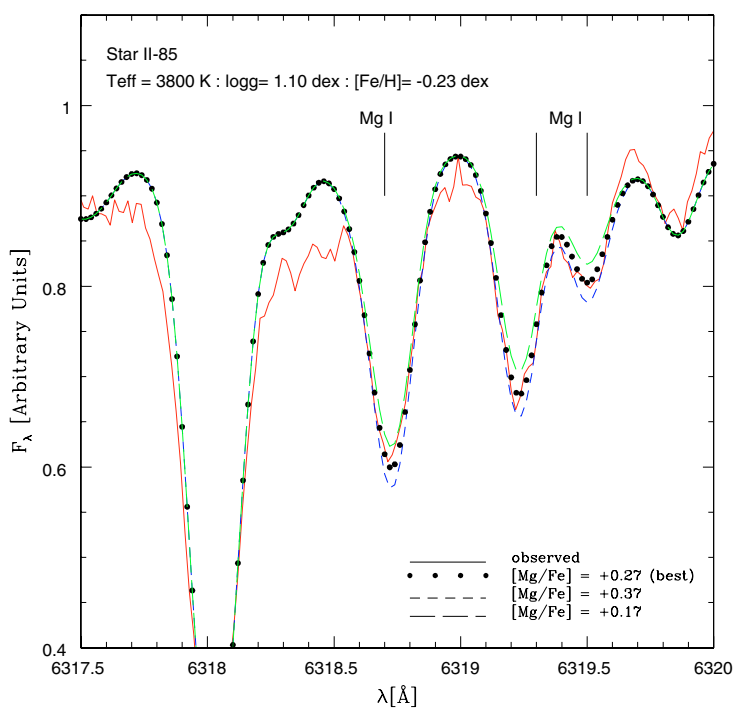

Fig. 4. Magnesium triplet at $6318.7,6319.2,6319.4 \AA$ in II-85 computed with $[\mathrm{Mg} / \mathrm{Fe}]=+0.27$ (dotted line, best fit), $[\mathrm{Mg} / \mathrm{Fe}]=+0.37$ (short-dashed line), and $[\mathrm{Mg} / \mathrm{Fe}]=+0.17$ (long-dashed line) .

gives $[\mathrm{Fe} \mathrm{I} / \mathrm{H}]=-0.22$ and $[\mathrm{Fe} \mathrm{I} / \mathrm{H}]=-0.21$ (except for star 267092, where $[\mathrm{Fe} \mathrm{I} / \mathrm{H}]=-0.19$ and $[\mathrm{Fe} \mathrm{II} / \mathrm{H}]=-0.46)$. In other words, the metallicities are the same whether they are computed with the photometric or the spectroscopic parameters, since the two sets of parameters are similar; and as demonstrated in Fig. 3, the spectroscopic parameters give a better agreement between the four stars than the photometric ones.

\subsubsection{Comparison with Barbuy et al. (1992)}

In Barbuy et al. (1992) a metallicity of $[\mathrm{Fe} / \mathrm{H}]=-0.20$ was found, in agreement with the present work. A reddening $E(B-V)=0.73$ and a photometric effective temperature was adopted. Despite the much lower $S / N$ of those spectra, the 




Fig. 5. Silicon line at $5948.548 \AA$ in II- 85 computed with $[\mathrm{Si} / \mathrm{Fe}]=$ +0.20 (dotted line, best fit), $[\mathrm{Si} / \mathrm{Fe}]=+0.30$ (short-dashed line), and $[\mathrm{Si} / \mathrm{Fe}]=+0.10$ (long-dashed line $)$.

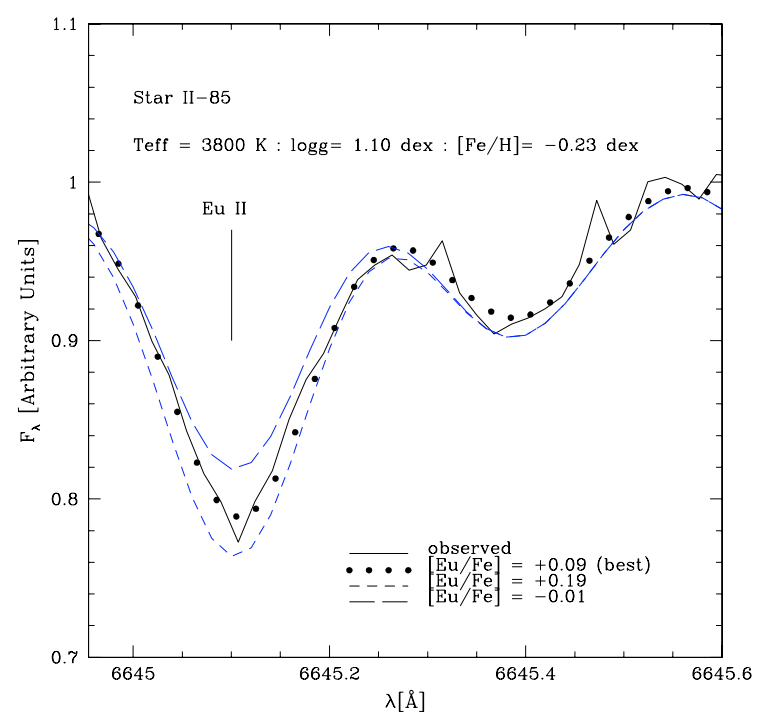

Fig. 6. Europium line at $6645 \AA$ in II-85 computed with $[\mathrm{Eu} / \mathrm{Fe}]=$ +0.09 (dotted line, best fit), $[\mathrm{Eu} / \mathrm{Fe}]=+0.19$ (short-dashed line), and $[\mathrm{Eu} / \mathrm{Fe}]=-0.01$ (long-dashed line) .

overall fit of synthetic spectra, which take blanketing lowering the continuum into account, proved to be a satisfactory method.

\subsubsection{Comparison with Barbuy et al. (1999)}

The atmospheric parameters adopted for II-85, the single star in common with the present work, were $T_{\text {eff }}=4000 \mathrm{~K}, \log g=$ $1.26(0.70), v_{\mathrm{t}}=1.30 \mathrm{~km} \mathrm{~s}^{-1}$ and $[\mathrm{Fe} / \mathrm{H}]=-0.50$. The VI colours obtained with HST (Ortolani et al. 1995) were used, which differ slightly from the WFI ones used in the present paper.

We found 3 main sources of uncertainty in the metallicity of $[\mathrm{Fe} / \mathrm{H}]=-0.55$ found in Barbuy et al. (1999), by comparing the parameters of star II-85 in common with the present work.

(i) The effective temperature of $4000 \mathrm{~K}$ was derived in Barbuy et al. (1999) using photometry, adopting a reddening of $E(B-V)=0.70$, whereas in the present work a spectroscopic excitation temperature of $3842 \mathrm{~K}$ is obtained.
Table 5. Sensitivity of abundances to changes in $\Delta T_{\text {eff }}=100 \mathrm{~K}$, $\Delta \log g=+0.3$, and $\Delta v_{\mathrm{t}}=0.2 \mathrm{~km} \mathrm{~s}^{-1}$. In the last column the corresponding total error is given.

\begin{tabular}{lcccc}
\hline \hline $\begin{array}{l}\text { Species } \\
(1)\end{array}$ & $\begin{array}{c}\Delta T \\
(2)\end{array}$ & $\begin{array}{c}\Delta \log g \\
(3)\end{array}$ & $\begin{array}{c}\Delta v_{\mathrm{t}} \\
(4)\end{array}$ & $\begin{array}{c}\left(\sum x^{2}\right)^{1 / 2} \\
(5)\end{array}$ \\
\hline & \multicolumn{5}{c}{$\mathrm{II}-64$} \\
\hline$[\mathrm{Na} \mathrm{I} / \mathrm{Fe}]$ & +0.08 & +0.02 & -0.02 & +0.08 \\
{$[\mathrm{Mg} \mathrm{I} / \mathrm{Fe}]$} & -0.01 & -0.02 & +0.01 & +0.02 \\
{$[\mathrm{Al} \mathrm{I} / \mathrm{Fe}]$} & +0.10 & +0.00 & +0.01 & +0.10 \\
{$[\mathrm{Si} \mathrm{I} / \mathrm{Fe}]$} & -0.10 & -0.01 & -0.05 & +0.11 \\
{$[\mathrm{Ca} / \mathrm{Fe}]$} & +0.05 & +0.01 & -0.15 & +0.16 \\
{$[\mathrm{Ti} \mathrm{I} / \mathrm{Fe}]$} & +0.10 & +0.03 & -0.10 & +0.14 \\
{$[\mathrm{Ti} \mathrm{II} / \mathrm{Fe}]$} & +0.05 & +0.20 & +0.05 & +0.21 \\
{$[\mathrm{Zr} \mathrm{I} / \mathrm{Fe}]$} & +0.20 & +0.01 & +0.01 & +0.20 \\
{$[\mathrm{Ba} \mathrm{II} / \mathrm{Fe}]$} & +0.01 & +0.05 & -0.20 & +0.21 \\
{$[\mathrm{La} \mathrm{II} / \mathrm{Fe}]$} & +0.01 & +0.05 & +0.02 & +0.05 \\
{$[\mathrm{Eu} \mathrm{II} / \mathrm{Fe}]$} & +0.01 & +0.06 & +0.01 & +0.06 \\
\hline$[\mathrm{Fe} \mathrm{I} / \mathrm{H}]$ & -0.03 & -0.04 & +0.07 & +0.09 \\
{$[\mathrm{Fe} \mathrm{II} / \mathrm{H}]$} & +0.12 & -0.11 & +0.06 & +0.17 \\
\hline
\end{tabular}

Table 6. Fe I lines used in the present work in common with Barbuy et al. (1999).

\begin{tabular}{ccccc}
\hline \hline$\lambda(\AA)$ & $\chi_{\mathrm{ex}}(\mathrm{eV})$ & $\log g f$ & Present & Barbuy et al. (1999) \\
\hline 5861.110 & 4.280 & -2.450 & 24.9 & 25. \\
6082.710 & 2.220 & -3.570 & 131.1 & 102. \\
6096.660 & 3.980 & -1.930 & 70.1 & 74. \\
6120.250 & 0.910 & -5.950 & 96.4 & 69. \\
6475.620 & 2.560 & -2.940 & 127.5 & 118. \\
6481.870 & 2.280 & -2.980 & 148.5 & 117. \\
6574.230 & 0.990 & -5.040 & 149.3 & 130. \\
6739.520 & 1.560 & -4.940 & 98.4 & 49. \\
\hline
\end{tabular}

(ii) The main source of discrepancy has been an inappropriate correction of gravity by 0.60 dex. The difference of $\log g=$ 1.26 found then from classical formulae and $\log g=0.70$ as adopted led to a $\Delta[\mathrm{Fe} / \mathrm{H}]=-0.3$.

(iii) Equivalent widths for the star II-85 for lines in common are compared in Table 6. It appears that in the early work of $1999, W_{\lambda}$ 's of strong lines were systematically lower by about $20 \mathrm{~m} \AA$, which leads to a metallicity that is lower by $\Delta[\mathrm{Fe} / \mathrm{H}] \approx 0.1 \mathrm{dex}$. The reason for the discrepancy in $W_{\lambda}$ 's comes from the lower quality of the 1999 CASPEC spectra both in resolution and $S / N$. This can be seen in Fig. 7, which compares the two sets of observations for the star II-85.

\subsubsection{Comparison with Cohen et al. (1999)}

The five stars of Cohen et al. were red horizontal branch stars with $T_{\text {eff }}=4630-4830 \mathrm{~K}, \log g=2.3, v_{\mathrm{t}}=1.4-2.5 \mathrm{~km} \mathrm{~s}^{-1}$, and a mean metallicity of $[\mathrm{Fe} \mathrm{I} / \mathrm{H}]=-0.16$ and $[\mathrm{Fe} \mathrm{II} / \mathrm{H}]=-0.18$. Effective temperatures were adopted from excitation equilibrium of Fe I lines. In order to be compatible with the colours, they deduced $E(B-V)=0.78$ for 3 stars and $E(B-V)=0.86$ for the other two stars. The metallicity is in good agreement with the present work, whereas there is some discrepancy concerning the abundance ratios, as shown in Table 7 . A difference $\Delta[\mathrm{Ca} / \mathrm{Fe}]=$ 0.2 is explained by an offset in the solar $\mathrm{Ca}$ abundance adopted (Table 7), whereas the oscillator strengths for the lines in common agree. No lines in common are available for comparing Ti oscillator strengths, but there is an offset in the solar abundance of $\Delta[\mathrm{Ti} / \mathrm{Fe}]=0.05$. Possible differences in damping 
Table 7. Comparison of solar abundances and abundance ratios obtained for NGC 6553 between the present work and Cohen et al. (1999).

\begin{tabular}{ccccc}
\hline \hline & \multicolumn{2}{c}{ Present work } & \multicolumn{2}{c}{ Cohen et al. (1999) } \\
Element & $\epsilon_{\odot}(\mathrm{X})$ & {$[\mathrm{X} / \mathrm{Fe}]$} & $\epsilon_{\odot}(\mathrm{X})$ & {$[\mathrm{X} / \mathrm{Fe}]$} \\
\hline $\mathrm{O}$ & 8.77 & +0.2 & 8.68 & +0.50 \\
$\mathrm{Mg}$ & 7.58 & +0.28 & 7.54 & +0.41 \\
$\mathrm{Si}$ & 7.55 & +0.21 & 7.54 & +0.14 \\
$\mathrm{Ca}$ & 6.36 & +0.05 & 6.16 & +0.26 \\
$\mathrm{Ti}$ & 5.02 & -0.01 & 4.98 & +0.19 \\
\hline
\end{tabular}
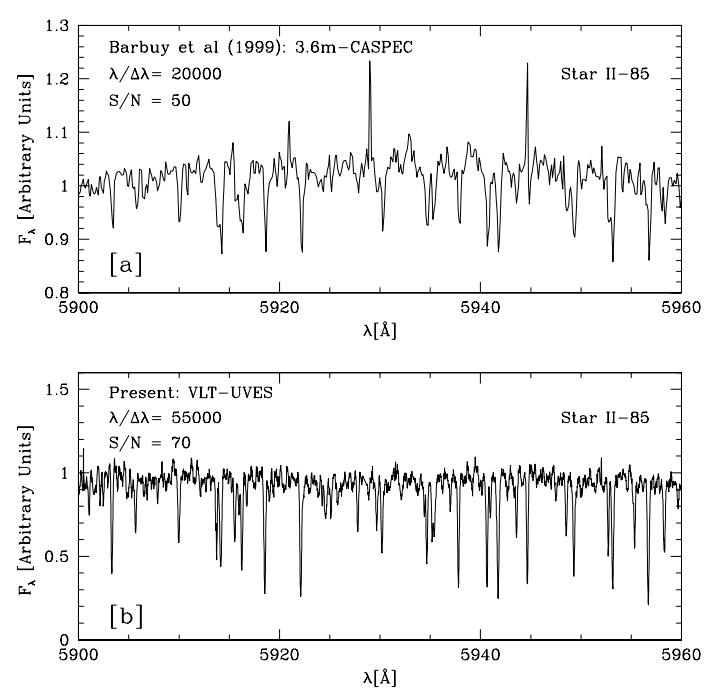

Fig. 7. Spectra of the giant II-85: comparison between the spectrum studied in Barbuy et al. (1999), obtained with the $3.6 \mathrm{~m}$ telescope and the CASPEC spectrograph $(R \sim 20000)$ and the present spectrum obtained with VLT-UVES $(R \sim 55000)$.

constants cannot be checked since they are not given in Cohen et al. (1999).

\subsubsection{Comparison with Origlia et al. (2002)}

Origlia et al. (2002) analysed 2 stars of $T_{\text {eff }}=4000 \mathrm{~K}, \log g=$ $1.0, v_{\mathrm{t}}=2 \mathrm{~km} \mathrm{~s}^{-1}$, and a mean metallicity of $[\mathrm{Fe} \mathrm{I} / \mathrm{H}]=-0.30$ was derived, together with enhancement of oxygen $[\mathrm{O} / \mathrm{Fe}]=+0.30$, and $[\alpha / \mathrm{Fe}]=+0.30(\mathrm{Mg}, \mathrm{Ca}$ and $\mathrm{Si})$. A reddening of $E(J-K)=$ 0.41 , compatible with $E(B-V)=0.70$, was used.

\subsubsection{Comparison with Meléndez et al. (2003)}

The parameters of II-85, again in common with the present work, of $T_{\text {eff }}=4000 \mathrm{~K}, \log g=1.2, v_{\mathrm{t}}=1.4 \mathrm{~km} \mathrm{~s}^{-1},[\mathrm{Fe} / \mathrm{H}]=-0.20$, found in Meléndez et al. (2003), show an effective temperature higher by $200 \mathrm{~K}$, in agreement with Barbuy et al. (1999). As in Barbuy et al. (1999), the VI colours from HST (Ortolani et al. 1995) were used, as well as a reddening of $E(B-V)=0.70$. Despite the difference with the present work, the resulting metallicities agree, which is explained by the low sensitivity of the high excitation potential infrared Fe I lines to $T_{\text {eff }}$ (see Table 6 in Meléndez et al. 2003).

\section{Abundance ratios}

\section{1. $\alpha$-elements $\mathrm{Mg}, \mathrm{Ca}, \mathrm{Si}, \mathrm{Ti}$}

The $\alpha$-elements provide information on the relative contributions of SNe type II and Ia in the enrichment of the interstellar medium

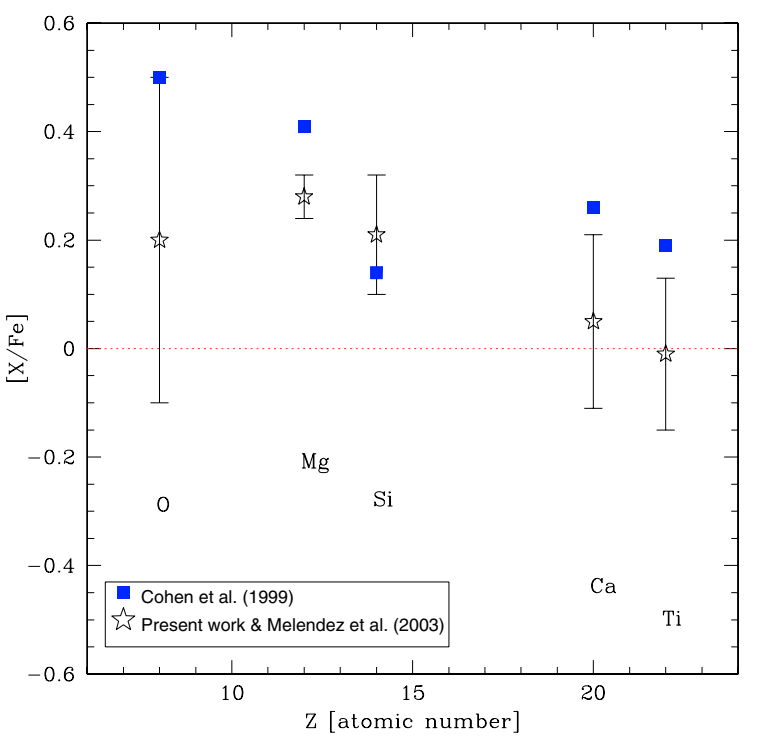

Fig. 8. Abundance pattern in $\alpha$-elements in NGC 6553 corresponding to: (i) a mean of the four sample stars derived in the present work and adopting oxygen from Meléndez et al. (2003) (open stars) and (ii) abundances of the same elements given in Table 5 of Cohen et al. (1999) (filled squares).

prior to the formation of cluster stars. Type II Supernovae are the main sources of $\alpha$-elements (Woosley \& Weaver 1995), the yields depending upon the mass of the progenitor.

Magnesium and silicon are moderately enhanced with $[\mathrm{Mg} / \mathrm{Fe}]=+0.28 \pm 0.04$ and $[\mathrm{Si} / \mathrm{Fe}]=+0.21 \pm 0.05$ dex. A solar calcium-to-iron $[\mathrm{Ca} / \mathrm{Fe}]=+0.05 \pm 0.09 \mathrm{dex}$ and titanium-to-iron $[\mathrm{Ti} \mathrm{I} / \mathrm{Fe}]=-0.01 \pm 0.14$ and $[\mathrm{Ti} \mathrm{II} / \mathrm{Fe}]=-0.02 \pm 0.06$ are found In Fig. $8 \alpha$-element abundances from the present work (Table 4), Meléndez et al. (2003), and Cohen et al. (1999) are shown as a function of Atomic Number $Z$. While in Cohen et al. (1999), $\mathrm{O}, \mathrm{Mg}, \mathrm{Si}, \mathrm{Ca}$, and $\mathrm{Ti}$ are enhanced, in the present work $\mathrm{Ca}$ and $\mathrm{Ti}$ are solar, whereas $\mathrm{Si}$ and $\mathrm{Mg}$ are enhanced. The high oxygen abundance in Cohen et al. (1999), relative to the value found by Meléndez et al. (2003), may be due to the use of the OI 771-7 nm triplet that systematically gives higher abundances (Kiselman 1995). Besides, there is an offset in the solar oxygen abundance adopted (Table 7 ) of $\Delta[\mathrm{O} / \mathrm{Fe}]=0.09$. The mean abundance of $[\mathrm{Ti} / \mathrm{Fe}]$ in NGC 6553 resembles the value found in Zoccali et al. (2004) for NGC 6528, obtained using the same atomic parameters.

\subsection{Odd-Z elements $\mathrm{Na}, \mathrm{Al}$}

Sodium and aluminum are odd-Z elements produced by neutron capture in the $\mathrm{Ne}-\mathrm{Na}$ and $\mathrm{Mg}-\mathrm{Al}$ cycles during carbon burning. A mean value of $[\mathrm{Na} / \mathrm{Fe}]=+0.16 \pm 0.23$ dex and $[\mathrm{Al} / \mathrm{Fe}]=$ $+0.18 \pm 0.06$ dex are found. No corrections for non-LTE effects were applied. The red giant II-85 of our sample is enhanced in $\mathrm{Na}$ by $[\mathrm{Na} / \mathrm{Fe}]=+0.34$ dex, while the other stars show solar ratios. This result is compatible with a mixing occurring along the RGB (Gratton et al. 2004), and to check this, it would be interesting to derive nitrogen abundances for our sample stars. Na is compatible with results by McWilliam \& Rich (1994, hereafter MR94); on the other hand, it is overabundant in NGC 6528 (Zoccali et al. 2004). A moderate enhancement of aluminum of $[\mathrm{Al} / \mathrm{Fe}]=+0.18$ is found. 


\subsection{Heavy elements $\mathrm{Zr}, \mathrm{Ba}, \mathrm{La}, \mathrm{Eu}$}

The s-process elements $\mathrm{Zr}, \mathrm{Ba}$, and $\mathrm{La}$ are produced through neutron-capture in asymptotic giant branch stars (Käppeler 1989; Busso et al. 1999). Abundances of the s- ( $\mathrm{Zr}$, Ba, and La) elements obtained were $[\mathrm{Zr} / \mathrm{Fe}]=-0.67 \pm 0.05,[\mathrm{Ba} / \mathrm{Fe}]=$ $-0.28 \pm 0.18$, and $[\mathrm{La} / \mathrm{Fe}]=-0.11 \pm 0.16$. Barium is depleted by -0.28 dex in NGC 6553, in contrast to an enhanced Ba abundance in 47 Tuc (Alves-Brito et al. 2005), a moderate enhancement in MR94, and a solar value in NGC 6528 (Zoccali et al. 2004). All s-elements are depleted. Edvardsson et al. (1993) show that $[\mathrm{Ba} / \mathrm{H}]$ is a better age indicator than $[\mathrm{Fe} / \mathrm{H}]$. Low s-element abundances suggest that the cluster is old, confirming the findings from CMDs (Ortolani et al. 1995).

The r-element europium abundance is $[\mathrm{Eu} / \mathrm{Fe}]=+0.10 \pm$ 0.02 dex. This moderate overabundance of Eu-to-Fe is compatible with those of the $\alpha$-elements, these elements being all produced by SNe II.

\section{Conclusions}

A detailed analysis based on high-resolution spectra for four giants of the metal-rich bulge globular cluster NGC 6553 was carried out. The present spectra are the highest resolution data so far for stars in this cluster. We find a mean metallicity value of $[\mathrm{Fe} / \mathrm{H}]=-0.20$, in agreement with Cohen et al. (1999) and Meléndez et al. (2003).

The $\alpha$-elements $\mathrm{Mg}$ and $\mathrm{Si}$ are overabundant relative to $\mathrm{Fe}$ with $[\mathrm{Mg} / \mathrm{Fe}]=+0.28$ dex and $[\mathrm{Si} / \mathrm{Fe}]=+0.21 \mathrm{dex}$, which might indicate a rapid chemical evolution history dominated by Type II Supernovae in the Galactic bulge. The lower abundances for the $\alpha$-elements $\mathrm{Ca}([\mathrm{Ca} / \mathrm{Fe}]=+0.05 \mathrm{dex})$ and $\mathrm{Ti}([\mathrm{Ti} / \mathrm{Fe}]=$ -0.01 dex) in stars of NGC 6553 might suggest a deficiency of low-mass Type II supernovae. The s-process elements $\mathrm{Zr}$, $\mathrm{Ba}$, and $\mathrm{La}$ are depleted by $[\mathrm{Zr} / \mathrm{Fe}]=-0.67 \mathrm{dex},[\mathrm{Ba} / \mathrm{Fe}]=$ -0.28 , and $[\mathrm{La} / \mathrm{Fe}]=-0.11 \mathrm{dex}$, respectively. The underabundance of s-elements is an independent indication that the cluster is old (Edvardsson et al. 1993). The r-process element Eu shows a small enhancement of $[\mathrm{Eu} / \mathrm{Fe}]=+0.10 \mathrm{dex}$, as do the $\alpha$-elements Ca and Ti. NGC 6553 appears to be very similar in its element abundance ratios to NGC 6528 analysed in Zoccali et al. (2004). The two clusters are very similar in age and metallicity, as pointed out in Ortolani et al. (1995).

Whether the metal-rich bulge clusters were formed during an early collapse or in merging events, as expected in hierarchical scenarios, can be revealed by abundance ratios. An early formation is accompanied by $\alpha$-enhancement. However in merging events, no detailed models are available: it is even possible that merging truncates star formation (Di Matteo et al. 2005). If instead bulge formation occurred via secular evolution of a bar (Kormendy \& Kennicutt 2004), solar abundance ratios would be expected, but this is not the case for NGC 6553. At the present time, it is important to gather metallicities and abundance ratios for other metal-rich clusters in the Galactic bulge, since their age spread and chemical abundances can constrain different models of bulge formation (Bekki 2005; Beasley et al. 2002; van den Bergh 1993).

Acknowledgements. A.A. acknowledges a Fapesp fellowship, No. 04/00287-9. B.B. and E.B. acknowledge grants from CNPq and Fapesp. D.M. and M.Z. acknowledge support from the FONDAP Centre for Astrophysics 15010003. This work benefited from the Latin American-European Network on Astrophysics and Cosmology (LENAC) of the European Union's ALFA Programme. This publication makes use of data products from the Two Micron All Sky Survey, which is a joint project of the University of Massachusetts and the Infrared Processing and Analysis Caltech, funded by the NASA and the NSF.

\section{References}

Alves-Brito, A., Barbuy, B., Ortolani, S., et al. 2005, A\&A, 435, 657 Alonso, A., Arribas, S., \& Martínez-Roger, C. 1998, A\&AS, 131, 209 Alonso, A., Arribas, S., \& Martínez-Roger, C. 1999, A\&AS, 140, 261 (AAM99) Alonso, A., Arribas, S., \& Martínez-Roger, C. 2001, A\&A, 376, 1039 Ashman, K. M., \& Zepf, S. E. 1992, ApJ, 384, 50

Ballester, P., Modigliani, A., Boitquin, O., et al. 2000, in The Messenger, 101, 31

Barbuy, B., Castro, S., Ortolani, S., \& Bica, E. 1992, A\&A, 259, 607

Barbuy, B., Bica, E., \& Ortolani, S. 1998, A\&A, 333, 117

Barbuy, B., Renzini, A., Ortolani, S., Bica, E., \& Guarnieri, M. D. 1999, A\&A, 341, 539

Barbuy, B., Perrin, M.-N., Katz, D., et al. 2003, A\&A, 404, 661

Barbuy, B., Zoccali, M., Ortolani, S., et al. 2006, A\&A, 449, 349

Beasley, M. A., Baugh, C. M., Forbes, D. A., Sharples, R. M., \& Frenk, C. S. 2002, MNRAS, 333, 383

Bekki, K. 2005, ApJ, 626, L93

Biehl, D. 1976, Ph.D. Thesis, University of Kiel

Brodie, J., \& Strader, J. 2006, ARA\&A, 44, 193

Busso, M., Gallino, R., \& Wasserburg, G. J. 1999, ARA\&A, 37, 239

Carpenter, J. M. 2001, AJ, 121, 2851

Carretta, E., Cohen, J. G., Gratton, R. G., \& Behr, B. B. 2001, AJ, 122, 1469

Cayrel, R., Perrin, M.-N., Barbuy, B., \& Buser, R. 1991, A\&A, 247, 108

Coelho, P., Barbuy, B., Perrin, M.-N., et al. 2001, A\&A, 376, 136

Côté, P. Marzke, R. O., \& West, M. J. 1998, ApJ, 501, 554

Cohen, J. G., Gratton, R. G., Behr, B. B., \& Carretta, E. 1999, ApJ, 523, 739

Cutri, R. M., Skrutskie, M. F., van Dyk, S., et al. 2003, VizieR On-line Data Catalog: II/246. Originally published in: University of Massachusetts and Infrared Processing and Analysis Center, (IPAC/California Institute of Technology)

Dean, J. F., Warpen, P. R., \& Cousins, A. J. 1978, MNRAS, 183, 569

Dekker, H., et al. 2000, SPIE, 4008, 534

Di Matteo, T., Springer, V., \& Hernquist, L. 2005, Nature, 433, 604

Dinescu, D. I., Girard, T. M., van Altena, W. F., \& López, C. E. 2003, AJ, 125, 1373

Edvardsson, B., Andersen, J., Gustafsson, B., et al. 1993, A\&A, 275, 101

Forbes, D., Brodie, J., \& Grillmair, C. 1997, AJ, 113, 1652

Gratton, R. G., Sneden, C., \& Carretta, E. 2004, ARA\&A, 42, 385

Grevesse, N., \& Sauval, J. N. 1998, SSRev, 35, 161

Guarnieri, M. D., Ortolani, S., Montegriffo, P., et al. 1998, A\&A, 331, 70

Harris, W. E. 1996, AJ, 112, 1487

Hartwick, F. D. A. 1975, PASP, 87, 77

Käppeler, F., Beer, H., \& Wisshak, K. 1989, Rep. Prog. Phys., 52, 945

Kiselman, D., \& Nordlund, A. 1995, A\&A, 302, 578

Kormendy, J., \& Kennicutt, R. C. Jr. 2004, ARA\&A, 42, 603

Lawler, J. E., Wickliffe, M. E., Hartog, A. D., \& Sneden, C. 2001, ApJ, 563, 1075

Martin, W. C., Fuhr, J. R., Kelleher, D. E., et al. 2002, NIST Atomic Database (version 2.0), http://physics.nist.gov/asd, National Institute of Standards and Technology, Gaithersburg, MD.

McWilliam, A., \& Rich, R. M. 1994, ApJS, 91, 749

Meléndez, J., \& Barbuy, B. 2002, ApJ, 575, 474

Meléndez, J., \& Barbuy, B. 2006, in preparation

Meléndez, J., Barbuy, B., Bica, E., et al. 2003, A\&A, 411, 417

Meléndez, J., Shchukina, N. G., Vasiljeva, I. E., \& Ramirez, I. 2006, ApJ, 642, 1082

Minniti, D. 1995, AJ, 109, 1663

Origlia, L., Rich, R. M., \& Castro, S. 2002, AJ, 123, 1559

Ortolani, S., Barbuy, B., \& Bica, E. 1990, A\&A, 236, 362

Ortolani, S., Renzini, A., Gilmozzi, R., et al. 1995, Nature, 377, 701

Plez, B., Brett, J. M., \& Nordlund, Å. 1992, A\&A, 256, 551

Rieke, G. H., \& Lebofsky, M. J. 1985, ApJ, 288, 618

Rutledge, G. A., Hesser, J. E., Stetson, P. B., et al. 1997, PASP, 109, 883

Sagar, R., Subramaniam, A., Richtler, T., \& Grebel, E. K. 1999, A\&AS, 135, 391

Spite, M. 1967, Ann. Astrophys., 30, 211

van den Bergh, S. 1993, ApJ, 411, 178

Woosley, S. E., \& Weaver, T. A. 1995, ApJS, 101, 181

Zoccali, M., Renzini, A., Ortolani, S., Bica, E., \& Barbuy, B. 2001, AJ, 125, 994 Zoccali, M., Renzini, A., Ortolani, S., Bica, E., \& Barbuy, B. 2003, AJ, 121, 2638

Zoccali, M., Barbuy, B., Hill, V., et al. 2004, A\&A, 423, 507 
A. Alves-Brito et al.: VLT-UVES abundance analysis of four giants in NGC 6553 , Online Material p 1

\section{Online Material}




\section{Appendix A: Line list}

Table A.1. Line list used for synthesis showing species, wavelength, excitation potential, damping constant, $g f$-values, and abundances derived line-by-line.

\begin{tabular}{|c|c|c|c|c|c|c|c|c|}
\hline \multirow[b]{2}{*}{ Species } & \multirow[b]{2}{*}{$\lambda(\AA)$} & \multirow[b]{2}{*}{$\chi_{\mathrm{ex}}(\mathrm{eV})$} & \multirow[b]{2}{*}{$C 6$} & \multirow[b]{2}{*}{$\log g f$} & \multicolumn{4}{|c|}{$\overline{[\mathrm{X} / \mathrm{Fe}]}$} \\
\hline & & & & & II-64 & II-85 & III-8 & 267092 \\
\hline \multicolumn{9}{|c|}{$\alpha$-elements } \\
\hline Mg I & 6318.720 & 5.110 & $0.30 \mathrm{E}-31$ & -2.100 & +0.27 & +0.27 & +0.27 & +0.12 \\
\hline Mg I & 6319.242 & 5.110 & $0.30 \mathrm{E}-31$ & -2.360 & +0.27 & +0.27 & +0.27 & +0.27 \\
\hline $\mathrm{Mg} \mathrm{I}$ & 6319.490 & 5.110 & $0.30 \mathrm{E}-31$ & -2.900 & +0.42 & +0.27 & +0.35 & +0.27 \\
\hline $\mathrm{Si} \mathrm{I}$ & 5948.548 & 5.082 & $2.19 \mathrm{E}-30$ & -1.170 & +0.20 & +0.20 & +0.25 & +0.15 \\
\hline Si I & 6142.490 & 5.620 & $0.30 \mathrm{E}-31$ & -1.580 & +0.20 & +0.12 & +0.10 & +0.20 \\
\hline Si I & 6145.020 & 5.610 & $0.30 \mathrm{E}-31$ & -1.500 & & +0.25 & & \\
\hline $\mathrm{Si} \mathrm{I}$ & 6155.020 & 5.620 & $0.30 \mathrm{E}-30$ & -0.850 & +0.40 & +0.30 & +0.25 & +0.20 \\
\hline Si I & 6243.823 & 5.610 & $0.30 \mathrm{E}-32$ & -1.300 & +0.35 & $\ldots$ & +0.10 & +0.15 \\
\hline Si I & 6244.480 & 5.610 & $0.30 \mathrm{E}-31$ & -1.270 & +0.30 & $\ldots$ & +0.15 & +0.15 \\
\hline Si I & 6414.987 & 5.870 & $0.30 \mathrm{E}-30$ & -1.128 & +0.20 & 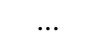 & +0.15 & +0.15 \\
\hline $\mathrm{Ca} \mathrm{I}$ & 6102.723 & 1.879 & $4.54 \mathrm{E}-31$ & -0.930 & +0.09 & +0.14 & +0.09 & -0.11 \\
\hline Ca I & 6156.030 & 2.521 & $4.00 \mathrm{E}-31$ & -2.590 & +0.14 & +0.09 & +0.09 & -0.01 \\
\hline Ca I & 6161.297 & 2.523 & $4.00 \mathrm{E}-31$ & -1.420 & +0.14 & +0.24 & +0.14 & -0.01 \\
\hline Ca I & 6162.173 & 2.523 & $3.00 \mathrm{E}-31$ & -0.090 & +0.09 & +0.09 & +0.14 & -0.01 \\
\hline Ca I & 6166.439 & 2.521 & $3.97 \mathrm{E}-31$ & -1.156 & +0.09 & +0.09 & -0.01 & -0.06 \\
\hline $\mathrm{Ca} \mathrm{I}$ & 6169.042 & 2.523 & $3.97 \mathrm{E}-31$ & -0.900 & +0.29 & +0.19 & +0.14 & -0.06 \\
\hline $\mathrm{Ca} I$ & 6169.563 & 2.526 & $4.00 \mathrm{E}-31$ & -0.630 & +0.29 & +0.19 & +0.14 & -0.11 \\
\hline $\mathrm{Ca} \mathrm{I}$ & 6439.075 & 2.526 & $3.40 \mathrm{E}-32$ & +0.300 & +0.14 & +0.09 & +0.14 & -0.11 \\
\hline Ca I & 6455.598 & 2.523 & $3.39 \mathrm{E}-32$ & -1.550 & +0.19 & +0.09 & +0.14 & +0.04 \\
\hline Ca I & 6464.679 & 2.520 & $3.40 \mathrm{E}-32$ & -2.480 & +0.24 & -0.06 & +0.24 & +0.24 \\
\hline $\mathrm{Ca} \mathrm{I}$ & 6471.662 & 2.526 & $3.39 \mathrm{E}-32$ & -0.800 & +0.09 & +0.19 & +0.09 & -0.21 \\
\hline $\mathrm{Ca} \mathrm{I}$ & 6493.788 & 2.526 & $3.37 \mathrm{E}-32$ & +0.000 & -0.11 & +0.19 & -0.21 & -0.21 \\
\hline Ca I & 6499.654 & 2.526 & $3.37 \mathrm{E}-32$ & -0.850 & -0.11 & +0.19 & -0.21 & -0.06 \\
\hline $\mathrm{Ca} \mathrm{I}$ & 6508.850 & 2.526 & $3.37 \mathrm{E}-32$ & -2.510 & +0.09 & -0.06 & -0.06 & -0.11 \\
\hline $\mathrm{Ca} \mathrm{I}$ & 6572.778 & 0.000 & $1.75 \mathrm{E}-32$ & -4.320 & -0.01 & $\cdots$ & -0.16 & -0.31 \\
\hline Ti I & 5866.452 & 1.067 & $2.16 \mathrm{E}-32$ & -0.840 & +0.18 & +0.28 & +0.13 & +0.03 \\
\hline Ti I & 5965.828 & 1.879 & $2.14 \mathrm{E}-32$ & -0.409 & +0.13 & +0.06 & +0.13 & -0.12 \\
\hline Ti I & 5978.543 & 1.873 & $2.14 \mathrm{E}-32$ & -0.496 & +0.18 & +0.06 & +0.08 & -0.12 \\
\hline Ti I & 6064.629 & 1.046 & $2.06 \mathrm{E}-32$ & -1.944 & +0.03 & +0.31 & -0.17 & -0.17 \\
\hline Ti I & 6091.174 & 2.267 & $3.89 \mathrm{E}-32$ & -0.423 & +0.03 & +0.26 & +0.03 & -0.12 \\
\hline Ti I & 6126.217 & 1.067 & $2.06 \mathrm{E}-32$ & -1.424 & +0.03 & +0.31 & +0.03 & -0.47 \\
\hline Ti I & 6258.110 & 1.440 & $4.75 \mathrm{E}-32$ & -0.360 & -0.07 & +0.11 & -0.17 & -0.52 \\
\hline Ti I & 6303.756 & 1.443 & $1.53 \mathrm{E}-32$ & -1.566 & +0.08 & +0.06 & -0.07 & -0.12 \\
\hline Ti I & 6312.237 & 1.460 & $4.75 \mathrm{E}-32$ & -1.552 & +0.08 & +0.11 & -0.07 & -0.07 \\
\hline Ti I & 6336.102 & 1.443 & $0.30 \mathrm{E}-31$ & -1.742 & -0.02 & +0.11 & -0.07 & -0.07 \\
\hline Ti I & 6508.153 & 1.430 & $1.46 \mathrm{E}-32$ & -2.050 & +0.03 & +0.11 & -0.07 & -0.07 \\
\hline Ti I & 6554.224 & 1.443 & $2.72 \mathrm{E}-32$ & -1.219 & +0.03 & +0.11 & -0.17 & -0.17 \\
\hline Ti I & 6556.062 & 1.460 & $2.74 \mathrm{E}-32$ & -1.075 & +0.03 & -0.04 & -0.17 & -0.22 \\
\hline Ti I & 6599.130 & 0.900 & $2.94 \mathrm{E}-32$ & -2.085 & -0.02 & +0.26 & -0.07 & -0.27 \\
\hline Ti I & 6743.124 & 0.900 & $0.30 \mathrm{E}-31$ & -1.628 & -0.07 & +0.26 & -0.07 & -0.07 \\
\hline Ti II & 6491.582 & 2.060 & $0.30 \mathrm{E}-31$ & -2.100 & +0.03 & +0.08 & -0.07 & -0.07 \\
\hline Ti II & 6559.576 & 2.050 & $0.30 \mathrm{E}-31$ & -2.480 & +0.09 & -0.07 & -0.02 & -0.07 \\
\hline \multicolumn{9}{|c|}{ Odd-Z elements } \\
\hline $\mathrm{Na} \mathrm{I}$ & 5682.647 & 2.102 & $0.30 \mathrm{E}-30$ & -0.700 & $\ldots$ & +0.34 & $\cdots$ & \\
\hline $\mathrm{Na} \mathrm{I}$ & 5688.217 & 2.102 & $0.30 \mathrm{E}-30$ & -0.457 & & +0.34 & $\ldots$ & \\
\hline $\mathrm{NaI}$ & 6154.225 & 2.102 & $0.90 \mathrm{E}-31$ & -1.570 & +0.12 & +0.34 & -0.08 & +0.12 \\
\hline $\mathrm{Na} \mathrm{I}$ & 6160.753 & 2.104 & $0.30 \mathrm{E}-31$ & -1.270 & +0.12 & $\ldots$ & -0.08 & +0.12 \\
\hline $\mathrm{Al} \mathrm{I}$ & 6696.032 & 3.143 & $0.30 \mathrm{E}-31$ & -1.481 & +0.28 & $\ldots$ & +0.28 & +0.13 \\
\hline Al I & 6698.667 & 3.143 & $0.30 \mathrm{E}-31$ & -1.782 & +0.23 & $\ldots$ & +0.03 & +0.13 \\
\hline \multicolumn{9}{|c|}{ Heavy elements } \\
\hline $\mathrm{ZrI}$ & 6143.216 & 0.070 & $0.30 \mathrm{E}-31$ & -1.100 & -0.74 & -0.64 & -0.64 & -0.64 \\
\hline Ba II & 6141.728 & 0.704 & & -0.070 & -0.08 & -0.28 & -0.58 & -0.38 \\
\hline Ba II & 6496.900 & 0.604 & & -0.380 & -0.08 & -0.08 & -0.38 & -0.38 \\
\hline La II & 6390.483 & 0.320 & & -1.410 & +0.01 & -0.22 & $\ldots$ & . \\
\hline Eu II & 6645.127 & 1.380 & & +0.120 & +0.09 & +0.09 & +0.10 & +0.14 \\
\hline
\end{tabular}


A. Alves-Brito et al.: VLT-UVES abundance analysis of four giants in NGC 6553 , Online Material p 3

Table A.2. Fe I and Fe II line list: (1) element; (2) wavelength; (3) lower excitation potential; (4) $\log g f$-values; (5)-(8) equivalent widths (mÅ).

\begin{tabular}{|c|c|c|c|c|c|c|c|}
\hline $\begin{array}{l}\text { Ion } \\
\text { (1) }\end{array}$ & $\begin{array}{c}\lambda(\AA) \\
(2)\end{array}$ & $\begin{array}{c}\chi_{\text {ex }}(\mathrm{eV}) \\
\text { (3) }\end{array}$ & $\begin{array}{c}\log g f \\
(4)\end{array}$ & $\begin{array}{c}\text { II-64 } \\
(5)\end{array}$ & $\begin{array}{c}\text { II-85 } \\
(6)\end{array}$ & $\begin{array}{c}\text { III-8 } \\
(7)\end{array}$ & $\begin{array}{c}267092 \\
(8)\end{array}$ \\
\hline \multicolumn{8}{|c|}{ Fe I } \\
\hline $\mathrm{Fe} \mathrm{I}$ & 5833.93 & 2.61 & -3.66 & & & 55.2 & $\ldots$ \\
\hline $\mathrm{Fe} \mathrm{I}$ & 5835.10 & 4.26 & -2.37 & 39.8 & 30.0 & $\ldots$ & $\ldots$ \\
\hline $\mathrm{Fe} \mathrm{I}$ & 5853.15 & 1.48 & -5.27 & 59.7 & 88.3 & 44.8 & 48.0 \\
\hline $\mathrm{Fe} \mathrm{I}$ & 5855.08 & 4.60 & -1.75 & 39.6 & 47.6 & 41.6 & 38.7 \\
\hline $\mathrm{Fe} \mathrm{I}$ & 5856.09 & 4.29 & -1.64 & 61.7 & 66.7 & 57.3 & 62.0 \\
\hline $\mathrm{Fe} \mathrm{I}$ & 5858.78 & 4.22 & -2.26 & 29.3 & 36.6 & 28.8 & 28.9 \\
\hline $\mathrm{Fe} \mathrm{I}$ & 5859.60 & 4.55 & -0.60 & 94.7 & 88.5 & 91.9 & 91.1 \\
\hline $\mathrm{Fe} \mathrm{I}$ & 5861.11 & 4.28 & -2.45 & 20.6 & 24.9 & 16.9 & 26.5 \\
\hline $\mathrm{Fe} \mathrm{I}$ & 5862.37 & 4.55 & -0.39 & 108.1 & 95.9 & 102.6 & 107.1 \\
\hline $\mathrm{Fe} \mathrm{I}$ & 5881.28 & 4.60 & -1.84 & & 34.8 & 38.0 & 35.1 \\
\hline Fe I & 5902.47 & 4.59 & -1.81 & 24.1 & 24.5 & 26.2 & 23.8 \\
\hline $\mathrm{Fe} \mathrm{I}$ & 5905.67 & 4.65 & -0.72 & 74.7 & 67.4 & 78.3 & 74.4 \\
\hline $\mathrm{Fe} \mathrm{I}$ & 5927.79 & 4.65 & -1.09 & 64.7 & 65.1 & 67.8 & 65.6 \\
\hline $\mathrm{Fe} \mathrm{I}$ & 5929.68 & 4.55 & -1.39 & 75.2 & 63.8 & 75.6 & 61.5 \\
\hline $\mathrm{Fe} \mathrm{I}$ & 5930.18 & 4.65 & -0.23 & 121.3 & 102.6 & 118.7 & 120.0 \\
\hline $\mathrm{Fe} \mathrm{I}$ & 5934.65 & 3.93 & -1.18 & 111.3 & 106.0 & 106.9 & 108.6 \\
\hline $\mathrm{Fe} \mathrm{I}$ & 5952.72 & 3.98 & -1.43 & 97.2 & 82.0 & 91.0 & 94.7 \\
\hline $\mathrm{Fe} \mathrm{I}$ & 5956.69 & 0.86 & -4.60 & 137.6 & $\ldots$ & 126.1 & 120.6 \\
\hline $\mathrm{Fe} \mathrm{I}$ & 5969.56 & 4.28 & -2.73 & & 15.8 & $\ldots$ & $\ldots$ \\
\hline $\mathrm{Fe} \mathrm{I}$ & 5983.69 & 4.55 & -0.78 & 97.5 & 83.4 & 81.8 & 91.6 \\
\hline $\mathrm{Fe} \mathrm{I}$ & 5987.07 & 4.79 & -0.45 & 93.2 & 84.7 & 92.0 & 95.9 \\
\hline $\mathrm{Fe} \mathrm{I}$ & 6003.01 & 3.88 & -1.11 & 119.7 & 106.9 & 119.2 & 113.9 \\
\hline $\mathrm{Fe} \mathrm{I}$ & 6024.06 & 4.55 & -0.11 & 124.8 & 107.7 & 121.0 & 119.1 \\
\hline Fe I & 6027.05 & 4.07 & -1.22 & 98.4 & 87.5 & 79.1 & 95.5 \\
\hline $\mathrm{Fe} \mathrm{I}$ & 6054.07 & 4.37 & -2.30 & 22.2 & 30.3 & 27.1 & 23.6 \\
\hline $\mathrm{Fe} \mathrm{I}$ & 6056.01 & 4.73 & -0.46 & 85.5 & 83.4 & 112.0 & 90.8 \\
\hline $\mathrm{Fe} \mathrm{I}$ & 6078.50 & 4.79 & -0.40 & 99.0 & 86.9 & 96.2 & 95.4 \\
\hline $\mathrm{Fe} \mathrm{I}$ & 6079.01 & 4.65 & -1.13 & 67.8 & 64.5 & 66.3 & 68.6 \\
\hline $\mathrm{Fe} \mathrm{I}$ & 6082.71 & 2.22 & -3.57 & 100.9 & 131.1 & 87.5 & 90.0 \\
\hline $\mathrm{Fe} \mathrm{I}$ & 6093.64 & 4.60 & -1.51 & 53.0 & 49.8 & 53.0 & 48.8 \\
\hline $\mathrm{Fe} \mathrm{I}$ & 6094.37 & 4.65 & -1.94 & 37.4 & 35.9 & 35.2 & 34.1 \\
\hline $\mathrm{Fe} \mathrm{I}$ & 6096.66 & 3.98 & -1.93 & 68.5 & 70.1 & 67.2 & 66.1 \\
\hline $\mathrm{Fe} \mathrm{I}$ & 6098.28 & 4.56 & -1.88 & 33.0 & 46.5 & 39.1 & 37.0 \\
\hline $\mathrm{Fe} \mathrm{I}$ & 6105.13 & 4.54 & -2.06 & 31.8 & 43.0 & 30.2 & 28.0 \\
\hline $\mathrm{Fe} \mathrm{I}$ & 6120.25 & 0.91 & -5.95 & 59.7 & 96.4 & 52.4 & 45.0 \\
\hline $\mathrm{Fe} \mathrm{I}$ & 6353.85 & 0.91 & -6.36 & & 71.7 & $\ldots$ & $\ldots$ \\
\hline $\mathrm{Fe} \mathrm{I}$ & 6392.54 & 2.28 & -4.03 & 71.7 & & 56.9 & 57.0 \\
\hline Fe I & 6419.95 & 4.73 & -0.25 & 107.5 & 97.8 & 101.5 & 105.1 \\
\hline $\mathrm{Fe} \mathrm{I}$ & 6475.62 & 2.56 & -2.94 & 121.3 & 127.5 & 110.5 & 103.3 \\
\hline $\mathrm{Fe} \mathrm{I}$ & 6481.87 & 2.28 & -2.98 & 141.2 & 148.5 & 121.1 & 123.1 \\
\hline $\mathrm{Fe} \mathrm{I}$ & 6498.94 & 0.96 & -4.70 & 140.4 & $\ldots$ & 124.6 & 126.6 \\
\hline $\mathrm{Fe} \mathrm{I}$ & 6518.37 & 2.83 & -2.75 & 105.0 & 120.1 & 102.8 & 105.8 \\
\hline $\mathrm{Fe} \mathrm{I}$ & 6533.93 & 4.56 & -1.45 & 69.5 & 58.5 & 61.3 & 66.8 \\
\hline $\mathrm{Fe} \mathrm{I}$ & 6556.81 & 4.79 & -1.68 & 24.3 & 28.4 & 30.0 & 25.6 \\
\hline $\mathrm{Fe} \mathrm{I}$ & 6569.22 & 4.73 & -0.42 & 105.5 & 102.0 & 100.2 & 101.6 \\
\hline $\mathrm{Fe} \mathrm{I}$ & 6574.23 & 0.99 & -5.04 & 114.3 & 149.3 & 102.3 & 98.5 \\
\hline $\mathrm{Fe} \mathrm{I}$ & 6575.02 & 2.59 & -2.82 & & $\ldots$ & 119.3 & 120.3 \\
\hline $\mathrm{Fe} \mathrm{I}$ & 6591.31 & 4.59 & -2.06 & 23.4 & $\ldots$ & 21.6 & 25.6 \\
\hline $\mathrm{Fe} \mathrm{I}$ & 6593.87 & 2.43 & -2.42 & $\ldots$ & $\ldots$ & 142.2 & 148.5 \\
\hline $\mathrm{Fe} \mathrm{I}$ & 6597.56 & 4.80 & -1.06 & 64.4 & 60.3 & 62.1 & 69.6 \\
\hline
\end{tabular}


A. Alves-Brito et al.: VLT-UVES abundance analysis of four giants in NGC 6553 , Online Material p 4

Table A.2. continued.

\begin{tabular}{lccccccc}
\hline \hline $\begin{array}{l}\text { Ion } \\
(1)\end{array}$ & $\begin{array}{c}\lambda(\AA) \\
(2)\end{array}$ & $\begin{array}{c}\chi_{\mathrm{ex}}(\mathrm{eV}) \\
(3)\end{array}$ & $\begin{array}{c}\log g f \\
(4)\end{array}$ & $\begin{array}{c}\text { II-64 } \\
(5)\end{array}$ & $\begin{array}{c}\text { II-85 } \\
(6)\end{array}$ & $\begin{array}{c}\text { III-8 } \\
(7)\end{array}$ & $\begin{array}{c}267092 \\
(8)\end{array}$ \\
\hline Fe I & 6608.03 & 2.28 & -4.04 & 70.5 & $\ldots$ & 59.7 & 56.6 \\
Fe I & 6699.14 & 4.59 & -2.18 & 29.4 & 25.8 & 24.7 & 20.7 \\
Fe I & 6703.57 & 2.76 & -3.15 & 93.4 & 96.5 & 82.6 & 84.5 \\
Fe I & 6705.11 & 4.61 & -1.40 & 77.2 & 58.5 & 72.7 & 74.2 \\
Fe I & 6710.32 & 1.48 & -4.87 & 94.6 & 112.1 & 83.1 & 79.3 \\
Fe I & 6713.74 & 4.80 & -1.60 & 40.0 & 33.1 & 36.7 & 37.9 \\
Fe I & 6715.38 & 4.59 & -1.64 & 65.3 & $\ldots$ & 57.6 & $\ldots$ \\
Fe I & 6725.36 & 4.10 & -2.30 & 41.1 & 41.5 & 39.2 & 41.5 \\
Fe I & 6726.67 & 4.59 & -1.15 & 71.5 & 56.4 & 67.6 & 66.7 \\
Fe I & 6733.15 & 4.64 & -1.58 & 48.7 & 54.6 & 52.8 & 50.4 \\
Fe I & 6739.52 & 1.56 & -4.94 & 72.2 & 98.4 & 64.0 & 57.2 \\
Fe I & 6752.71 & 4.64 & -1.37 & 74.7 & $\ldots$ & 66.4 & 62.8 \\
& & & Fe II & & & & \\
Fe II & 5991.36 & 3.15 & -3.54 & $\ldots$ & $\ldots$ & 43.4 & $\ldots$ \\
Fe II & 6084.09 & 3.20 & -3.79 & 31.2 & $\ldots$ & 31.7 & $\ldots$ \\
Fe II & 6149.24 & 3.89 & -2.69 & $\ldots$ & $\ldots$ & 51.4 & $\ldots$ \\
Fe II & 6247.56 & 3.89 & -2.30 & $\ldots$ & $\ldots$ & 50.7 & 54.7 \\
Fe II & 6432.68 & 2.89 & -3.57 & 42.0 & 29.1 & 53.8 & 55.9 \\
Fe II & 6456.39 & 3.90 & -2.05 & 69.3 & 33.5 & 72.2 & 64.5 \\
Fe II & 6516.08 & 2.89 & -3.31 & 66.9 & $\ldots$ & $\ldots$ & 69.3 \\
\hline
\end{tabular}

